LETTER TO THE EDITOR

\title{
Dziakova M
}

Department of Physiatry, Balneology and Medical Rehabilitation, University Hospital L. Pasteur, Kosice, Slovakia. miriam.dziakova@unlp.sk

Text in PDF www.elis.sk.

To the Editor:

After reading CLINICAL STUDY Impedance therapy in rehabilitation of degenerative disc disease, which was published in Bratislava Medical Journal 2020; 121 (2) we must respond to several facts stated there. For example: the authors chose the assessment of the volume of the intervertebral disc as a decisive criterion, but did not take into account many other existing factors that affect its changes $(1,2,3)$, some authors therefore recommend modification of the MRI examination (4) and therefore we consider the volume assessment alone to be an insufficient criterion. The authors did not take into account the affection of pain by alleviating, adjusting muscle imbalance (5), thus the increase in platelet volume in the patients of this study can hardly be explained as the cause of pain relief. The theoretical interpretation of the observed "Discgrowup" phenomenon is disputable. We consider the absence of parameters of the Specific Electrotherapeutic Impulse to be a relevant shortcoming of the work, and without them it is scientifically inadequate to publish the given work according to us. When combining SEP with a dry needle, the authors do not exclude the impact of the therapeutic effect of dry needle spraying, which could have influenced the final effect of the treatment. We consider the methods for the assessment of the effectiveness of the applied electrotherapeutic procedure to be insufficient. The authors did not select any scale for disability assessment, which is very important for the assessment of the output of a similar study. They do not report any musculoskeletal examination results except for the "joint range", which is not a standard examination, and the results reported by them do not belong to the rating scale used in the field of physiatry, balneology and medical rehabilitation. The not appropriate qualification of the authors in therapeutic procedures in the field of physiatry, balneology and medical rehabilitation can be the cause of serious shortcomings in rehabilitation treatment, as they state it, or more precisely do not state it at all. Such vaguely defined treatment method cannot be evaluated in any study.

The idea of inducing intervertebral disc growth with physical therapy has been discussed for a long time and is still a major challenge nowadays. However, influencing degenerative processes in the area of intervertebral discs and their objectification must be comprehensive and clearly defined.

\section{References}

1. Botsford DJ, Esses SI, Ogilvie-Harris DJ. In vivo diurnal variation in intervertebral disc volume and morphology. Spine (Phila Pa 1976) 1994; 19 (8): 935-940. DOI:10.1097/00007632-199404150-00012.

2. Hutton WC, Malko JA, Fajman WA. Lumbar disc volume measured by MRI: effects of bed rest, horizontal exercise, and vertical loading. Aviat Space Environ Med 2003; 74 (1): 73-78.

3. Kingsley MI, D'Silva LA, Jennings C, Humphries B, Dalbo VJ, Scanlan AT. Moderate-intensity running causes intervertebral disc compression in young adults. Med Sci Sports Exerc 2012; 44 (11): 2199-2204. DOI:10.1249/MSS.0b013e318260dbc1.

4. Torén L, Hebelka H, Kasperska I, Brisby H, Lagerstrand K. With axial loading during MRI diurnal T2-value changes in lumbar discs are neglectable: a cross sectional study. BMC Musculoskelet Disord 2018; 19 (1): 25. DOI:10.1186/s12891-018-1930-0.

5. Danneels L et al. The effect of experimental low back pain on lumbar muscle activity in people with a history of clinical low back pain: a muscle functional MRI study. J Neurophysiol 2016; 115 (2): 851-857. 